\title{
Role of Catalyst on the Formation of Resorcinol-Furfural Based Carbon Aerogels and Its Physical Properties
}

\author{
Karumully S. Rejitha, Poovakulathu A. Abraham, Narayana P. R. Panicker, \\ Kollanoor S. Jacob, Nimai C. Pramanik ${ }^{*}$ \\ Aerogel Laboratory, Centre for Materials for Electronics Technology (C-MET), \\ Thrissur, India \\ Email: "pramanik@cmet.gov.in,nc.pramanik@yahoo.com
}

Received December 10, 2012; revised January 14, 2013; accepted January 22, 2013

Copyright (C) 2013 Karumully S. Rejitha et al. This is an open access article distributed under the Creative Commons Attribution License, which permits unrestricted use, distribution, and reproduction in any medium, provided the original work is properly cited.

\begin{abstract}
Carbon aerogels (CAG) were synthesized by the pyrolysis of resorcinol-furfural based organic aerogels, derived from sol-gel polymerization of resorcinol and furfural using different catalysts followed by supercritical drying of as-prepared gels. Different catalysts viz. hydrochloric acid (HA), acetic acid (AcH) and hexamethylenetetramine (HMTA) of different concentrations were used for this purpose in order to study the role of different catalysts and the effect of R/C ratio (reactant to catalyst molar ratio) on the formation of organic gel monolith and their physical properties were investigated. Aerogels were thoroughly characterized by using CHN, FTIR, TG-DSC, XRD and SEM. A considerable reduction of gelation time and the formation of relatively denser organic gel were observed in the case of HMTA, which indicated the dual role (catalyst \& cross-linking agent) of HMTA during the polymerization/polycondensation of resorcinol and furfural. Carbon aerogels obtained by using different catalysts showed BET surface area, average pore size, total pore volumes in the range of $438-496 \mathrm{~m}^{2} / \mathrm{g}, 17.9-22.4 \AA$ and $0.20-0.27 \mathrm{~cm}^{3} / \mathrm{g}$, respectively. The SEM images and results revealed the presence of different morphologies of carbon aerogels, obtained by using different catalysts. The HMTA catalyzed samples were found to have highest surface area with particles in smaller in size and well interconnected 3D carbon network.
\end{abstract}

Keywords: Carbon Aerogel; Supercritical Drying; Sol-Gel Synthesis; Role of Catalyst; Surface Area Analysis; Pore Characteristics

\section{Introduction}

Carbon aerogels are promising materials for gas storage, hydrogen gas in particular and hence their synthesis has received much attention recently [1-4], since hydrogen is supposed to be the future fuel for the next generation and has the potential to replace the conventional energy sources like coal and petroleum. For specific usage of hydrogen, fuel cells in particular, its storage is the main concern and has become challenging topic of current research $[5,6]$. Out of several materials/techniques, such as pressure compression, liquefaction of gaseous $\mathrm{H}_{2}$, metal hydrides and inter metallic compounds, nanostructured carbon like carbon nanotube (CNT), graphite nanofiber (GNF), carbon aerogel (CAG), organometallic complexes, etc., have been investigated so far, and carbonaceous materials showed promising results [1-4,7-9]. Because of several outstanding physical properties of CAG,

"Corresponding author. such as large specific surface area, high porosity, tunable pore sizes, light weight, etc., it possess more attraction to the researchers for gas storage applications. Apart from gas storage applications, CAG also have several attractive applications, such as active electrode material for supercapacitors, batteries, fuel cells, gas filtration membranes, reinforcing agents for natural rubber, light weight structural composites for space explorations, and so on $[10,11]$. All these applications strongly depend on the structural and physico-chemical properties of the carbon aerogel and therefore, tuning of these properties through judicious selection of precursor materials and controlling of synthetic parameters are very critical for specific applications. It would be noteworthy to mention here that the tuning of these properties is possible by controlling the gel synthetic conditions, viz., precursor composition, nature of catalysts, presence of surface active agents (surfactants), drying procedure, etc., which have pronounced influence on the physical and chemical charac- 
teristics of final product (carbon aerogel) [12,13]. Several reports are known for the synthesis of carbonaerogel from resorcinol-formaldehyde based organic gels $[11,12$, 14-16]. Most of the reports described the various synthetic aspects of aerogel preparation and their physical properties. However, the preparation of carbon aerogel from other precursors, resorcinol-furfural is scanty [13, 17] and needs extensive study in order to use them as efficient hydrogen storage material. The properties like surface area, pore diameter, particle size etc. of the carbon aerogels are important properties, which can be controlled by the initial reactions conditions such as nature/concentration of catalyst and dilution during the organic gel formation. Even though numerous reports were published in regard to effect of catalyst concentrations on the final properties of carbon aerogel, the effect of different catalyst like $\mathrm{HCl}$, acetic acid, and hexamethylene-tetramine demands detailed investigations. In addition, conventionally the low temperature supercritical drying $\left(\mathrm{CO}_{2}\right.$ extraction) technique is used to obtained organic aerogels, which is known to be a lengthy process and also require extensive solvent exchange operation. A comparatively easy supercritical drying (SCD) process was established and adopted in this study for obtaining organic aerogels, which is faster and do not involve rigorous exchange of pore liquid. In this context, this paper describes the synthesis of carbon aerogel from resorcinol-furfural precursor by using different catalysts through faster supercritical drying process. The effects of various catalysts and the amount of catalysts on the formation of organic gel network as well as their physical properties were studied and also reported in this paper.

\section{Experimental}

\subsection{Materials}

Resorcinol (1,3 dihydroxy benzene, 99\%), furfural (98\%), hydrochloric acid $(\mathrm{HCl}, 0.1 \mathrm{M})$, glacial acetic acid $(\mathrm{AcH}$, $0.1 \mathrm{M}$ ), hexamethylenetetramine (HMTA, $0.1 \mathrm{M}$ ) and isopropyl alcohol (IPA, AR grade) were purchased from Merck India, Mumbai. All other chemical and solvents were AR grade commercial materials and were used asreceived without further purification.

\subsection{Synthesis of Resorcinol-Furfural Aerogel and Carbon Aerogel}

Resorcinol-furfural (referred as RFL) aerogels were prepared by sol-gel polymerization/condensation of resorcinol (referred as R) and furfural (referred as FL) in presence of catalyst in IPA medium followed by supercritical drying (above the critical conditions of IPA). Different catalyst such as $\mathrm{HCl}, \mathrm{CH}_{3} \mathrm{COOH}$, HMTA were used for the synthesis of RFL gels and several RFL gels were prepared by following a general procedure using $\mathrm{R} / \mathrm{FL}$ molar ratio of $1: 2$ and different amount of catalysts refer as R/C ratio in the range of $50-1000$ as described below.

Desired amount of catalyst solution was added to the mixture of resorcinol and furfural in IPA and the reaction mixture was stirred vigorously at $70^{\circ} \mathrm{C}$ under refluxing condition. Reaction mixture was then transferred into the gel chamber and kept aside for gelation at $70^{\circ} \mathrm{C}$. Dark brown color organic gel was formed after 2 - 5 days, which was then dried under the supercritical drying conditions of IPA using high pressure high temperature autoclave (Parr Instruments, USA) in order to obtain corresponding RFL aerogel. Sample obtained by using different catalysts were labeled as RFL-H, RFL-A and RFL-HM for $\mathrm{HCl}$, acetic acid and HMTA respectively.

Carbon aerogels (CAG) of corresponding precursors were obtained by the pyrolysis of respective RFL aerogel at $950^{\circ} \mathrm{C}$ under $\mathrm{N}_{2}$ atmosphere. CAG sample were then crushed, ball milled and sieved through $105 \mu \mathrm{m}$ mesh and labeled as CAG-H, CAG-A and CAG-HM as obtained from their respective precursors.

\subsection{Characterization \& Physical Measurements}

Chemical composition of RFL aerogels were studied by using Vario E1-III CHNS analyzer and Perkin-ElmerSpectrum-400 FTIR coupled with Pike-Gladi ATOR ATR attachments. FTIR spectra were recorded in the range of $400-4000 \mathrm{~cm}^{-1}$. Crystal structure of carbon aero-gel samples were studied using Bruker D-5005 X- ray powder diffractometer. Physical properties of aerogels were studied by $\mathrm{N} 2$ gas sorption analysis by using BET analyzer (NOVA 1200 Series, Quantachrome, USA). The gas sorption analyses (BET) were performed by using high purity nitrogen gas under liquid nitrogen temperature $(77 \mathrm{~K})$ and samples were degassed at $150^{\circ} \mathrm{C}$ for $3 \mathrm{~h}$ prior to the analysis. The Barrett-Joyner-Halenda (BJH) method was used for analysis of pore size distribution. Morphologies of different carbon aerogels were studied using JEOL JSM 5600 scanning electron microscopy (SEM).

\section{Results and Discussion}

\subsection{Synthesis and Characterization of Aerogels}

Resorcinol-furfural aerogels were prepared from resorcinol and furfural in presence of catalyst followed by supercritical drying of alcogels. Electrophilic aromatic substitution at its 2, 4 and 6 ring position of resorcinol allows the linkage with furfural leading to the formation of polymeric clusters having the high cross-linking densities. Consequently the condensation reaction in resorcinol-furfural precursors generates a three dimensional network via the formation of methylene and methylene ether bridges [13]. During the chemical reaction, furfural first forms a carbocation in presence of acid catalyst, which further reacts with electron rich carbon centre of 
resorcinol ring, yielding - $\mathrm{CH}(\mathrm{R})-\mathrm{O}-\mathrm{C}\left(\mathrm{R}^{\prime}\right) \mathrm{H}$-bridged polymers (where $R$ and $R^{\prime}$ is the resorcinol and furfural ring respectively). Formation of carbonium ion is therefore necessarily the first step for this polymerization reaction and the reaction between furfural and substituted resorcinol $\left(-\mathrm{CH}_{2} \mathrm{OH}\right.$ groups) produces nanometer sized clusters by inter/intra cross-linking, whose size is generally governed by the nature/concentration of the catalyst. As the poly-condensation reaction proceeds, the colour of the gel changes from pale brown to dark brown. Entire reactions are found to have strong catalyst dependency (both nature of catalyst and its conc.). The base catalyst (say, HMTA) first deprotonates the $\mathrm{OH}$ group of the re-

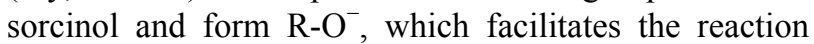
between the aromatic rings and furfural.

It is interesting to note that at the early stage of formation, the clusters may not be inter connected and hence, sometimes it leads to the formation of colloidal suspension, which collide through Brownian motion and start aggregation. Such aggregation finally leads to the formation of relatively larger clusters. Figure 1 shows the effect of various catalysts and their amount on the formation of monolithic organic gels. It was observed that the time required for the formation of RFL cluster is faster in the case of high catalyst concentration (i.e., low $\mathrm{R} / \mathrm{C}$ ratio), which was evidenced by the plot of gelation time vs. $\mathrm{R} / \mathrm{C}$ ratio (Figure 1). It was observed that the gelation time increased with decreasing the catalyst amount (increasing the $\mathrm{R} / \mathrm{C}$ ratios), irrespective of nature of catalyst and HMTA showed fastest the gelation as compared to that of the other catalysts. This might be due to the fact that HMTA act as catalyst as well as cross linking agent on the formation of RFL monolithic gel. As stated earlier, the size of primary organic clusters depends on the reaction mechanism, which are governed by the nature \& conc. of catalyst used. On the other hand, growth of clusters and formation of gel network structure depends on the rate of condensation, which are generally depends on the nature of functional group and the condensing agents, if any. Because of dual functionality of HMTA, HMTA enhanced the formation of alco-gel network via the stacking of organic clusters $[12,13,17]$.

Chemical compositions of different RFL aerogels were studied by elemental analyses in order to understand the presence of total carbon that formed the gel network. Results of elemental analysis are given in Table 1. It was observed that RFL aerogel, obtained from HMTA consists of relatively high amount of carbon, which indicated that HMTA produced relatively more cross-linked gel network. The similar trend of having high carbon was also observed in the case of corresponding CAG samples obtained by using HMTA.

The chemical environment of RFL aerogels were studied by using FTIR and the spectra were recorded

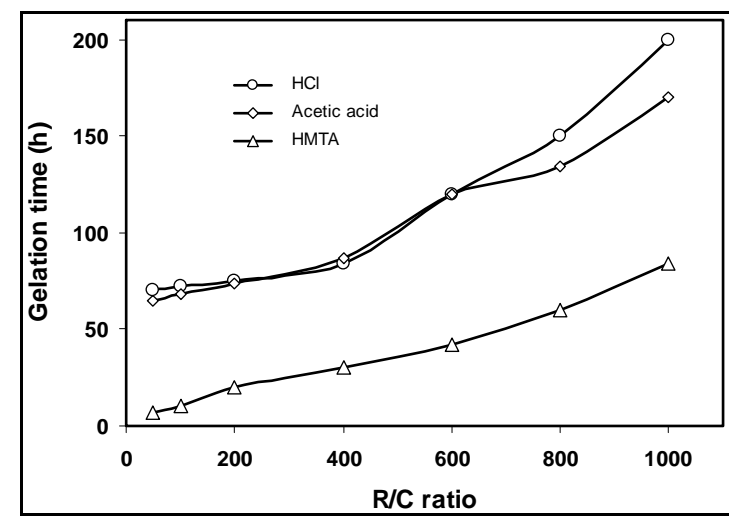

Figure 1. Plot of R/C ratio vs. gelation time for the formation of RFL gels under different catalysts.

Table 1. Results of elemental analysis of RFL aerogels and corresponding carbon aerogels.

\begin{tabular}{|c|c|c|c|c|}
\hline $\begin{array}{l}\text { Sample } \\
\text { code }\end{array}$ & Nature of aerogel & Catalyst used & $\% \mathrm{C}$ & $\% \mathrm{H}$ \\
\hline RFL-H & $\begin{array}{l}\text { Precursor organic } \\
\text { aerogel }\end{array}$ & $\begin{array}{l}\text { Hydrochloric } \\
\text { acid }\end{array}$ & 71.83 & 3.506 \\
\hline RFL-A & $\begin{array}{l}\text { Precursor organic } \\
\text { aerogel }\end{array}$ & Acetic acid & 74.47 & 3.854 \\
\hline RFL-HM & $\begin{array}{l}\text { Precursor organic } \\
\text { aerogel }\end{array}$ & $\begin{array}{l}\text { Hexamethylene } \\
\text { tetramine }\end{array}$ & 76.96 & 3.617 \\
\hline CAG-H & Carbon aerogel & $\begin{array}{l}\text { Hydrochloric } \\
\text { acid }\end{array}$ & 85.94 & 1.452 \\
\hline CAG- A & Carbon aerogel & Acetic acid & 86.76 & 1.549 \\
\hline CAG-HM & Carbon aerogel & $\begin{array}{l}\text { Hexamethylene } \\
\text { tetramine }\end{array}$ & 88.88 & 1.514 \\
\hline
\end{tabular}

from $400-4000 \mathrm{~cm}^{-1}$. FTIR spectra of RFL aerogel displayed several characteristic peaks, which were assigned to their respective vibrations. Three strong vibrations, observed at 1695,1606 , and $1469 \mathrm{~cm}^{-1}$, were assigned to the stretching vibrations of $>\mathrm{C}=\mathrm{O}$, aromatic ring, $\mathrm{O}-\mathrm{CH}_{2}$ and $\mathrm{C}-\mathrm{CH}_{2}-\mathrm{C}$ respectively. The peak, observed at 1440 $\mathrm{cm}^{-1}$ was assigned to the vibration due to $\mathrm{C}-\mathrm{H}$ group of $-\mathrm{CH}_{2} \mathrm{O}$-linkage. The vibrations at 960, 737, 803 and 839 $\mathrm{cm}^{-1}$ were assigned to the out of plane bending of $-\mathrm{C}-\mathrm{H}-$. Vibrations associated with the symmetric stretching of -C-O-C- and $\mathrm{O}-\mathrm{H}$ group of $\mathrm{CH}_{2} \mathrm{OH}$ were also observed at 1110 and $1020 \mathrm{~cm}^{-1}$ [18]. The relative intensity of the bands, observed at $1690 \mathrm{~cm}^{-1}$ (assigned to $>\mathrm{C}=\mathrm{O}$ ) was found to be high for RFL-A (acetic acid catalyzed sample) as compared to that of other samples, which might be due to the combined effect of $>\mathrm{C}=\mathrm{O}$ stretching frequency of the acetic acid and the furfural group.

\subsection{Study of Crystal Structure of RFL Derived Carbon Aerogel by X-Ray Diffraction}

Structure of different CAG, obtained from corresponding RFL precursors were evaluated by using powder XRD and the XRD patterns are shown in Figure 2. Although 


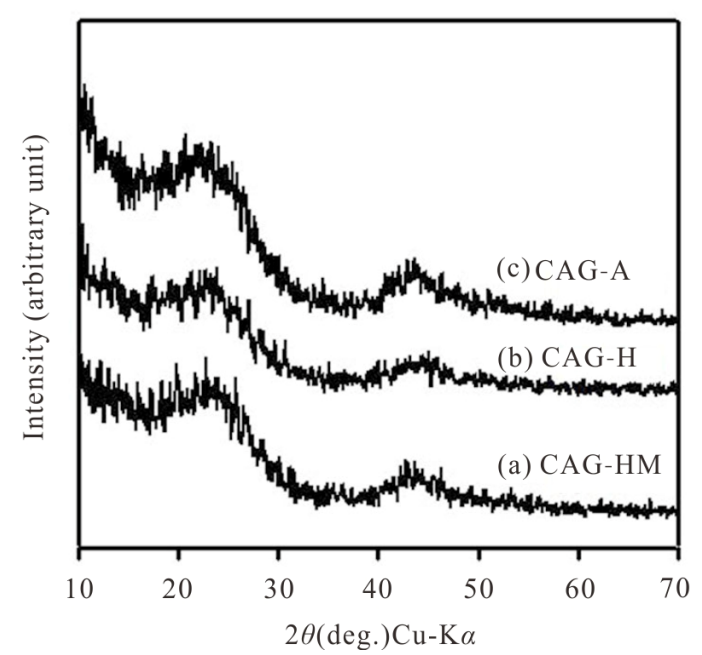

Figure 2. X-Ray diffraction pattern of different CAG samples obtained by using different catalysts: (a) HMTA, (b) $\mathrm{HCl}$ and (c) acetic acid.

the XRD patterns of different CAG samples were found to be almost identical, but displayed two broad peaks, centered at $2 \theta=23^{\circ} \& 43^{\circ}$. These reflections correspond to the graphitic phase of carbon (JCPDS\#22-1069). Lattice parameters for the reflections are also calculated from the XRD results and they are $\mathrm{a}=8.886 \pm 0.04 \AA$ and $\mathrm{c}=14.086 \pm 0.24 \AA$, which is found to be close to that of the reported values $(\mathrm{a}=8.946 \AA$ and $\mathrm{c}=14.078 \AA$, JCPDS\#22-1069). This indicates that carbon in carbon aerogels are crystalline material and have graphitic structure.

Figure 3 shows the SEM images of different carbon aeroges, obtained by using different catalysts. The morphologies of CAG particles were found to be nearly spherical in shape and they were interconnected. Size of CAG particles in the case of acid catalyzed species was found to be bigger and non-uniform. On the other hand, size of CAG particle obtained from HMTA source was relatively smaller and were more interconnected. This might be due to more cross-linked structure caused by the higher cross-linking ability of HMTA $[13,17]$ and gives rise to the formation of smaller particles.

\subsection{Studies of Physical Properties of RFL Derived Carbon Aerogels}

Physical properties, especially the surface characteristics of resorcinol-furfural derived carbon aerogels were evaluated by nitrogen gas sorption studies using BET analyzer at $77 \mathrm{~K}$. Results of BET analysis are given in Table 2. Carbon aerogels showed BET surface area (BET-SA) and total pore volume (TPV) in the range of $438-496$ $\mathrm{m}^{2} / \mathrm{g}$ and $0.20-0.27 \mathrm{~cm}^{3} / \mathrm{g}$ respectively. The micro-pore areas (MPA) and micro-pore volume (MPV) of carbon aerogels, obtained by using different catalysts were in the
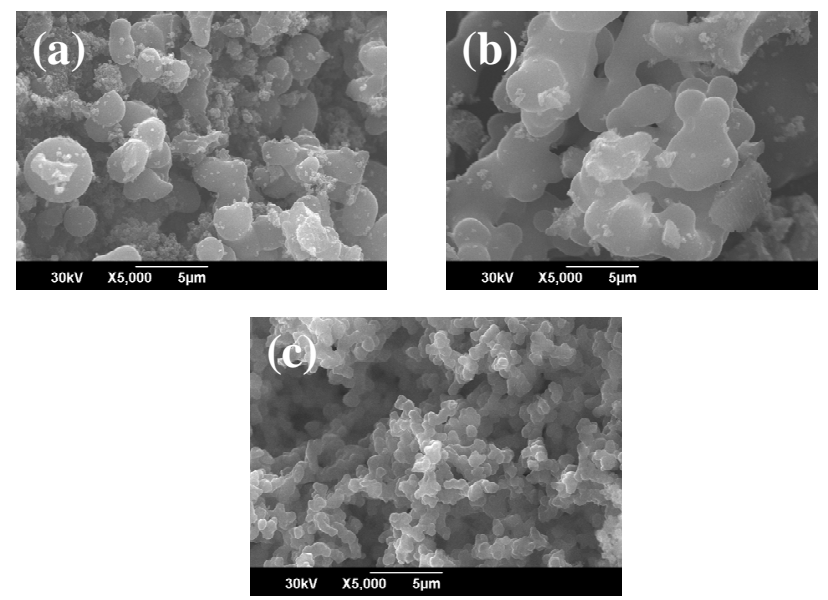

Figure 3. SEM images of carbon aerogels prepared from (a) HCl (b) acetic acid and (c) HMTA.

Table 2. BET surface area $\left(S A_{\mathrm{BET}}\right)$, external surface area (SA $\left.A_{\text {Ext. }}\right)$, micro-pore area (MPA), average pore size (APS), micro-pore volume (MPV) and total pore volume (TPV) of different CAG samples, obtained by using different catalysts.

\begin{tabular}{ccccccc}
\hline \multirow{2}{*}{$\begin{array}{c}\text { Sample } \\
\text { code }\end{array}$} & \multicolumn{2}{c}{ Surface area $\left(\mathrm{m}^{2} / \mathrm{g}\right)$} & \multicolumn{2}{c}{$\begin{array}{c}\text { Pore volume } \\
\left(\mathrm{cm}^{3} / \mathrm{g}\right)\end{array}$} & APS $(\AA)$ \\
\cline { 2 - 6 } & $\mathrm{SA}_{\mathrm{BET}}$ & $\mathrm{SA}_{\mathrm{Ext}}$ & $\mathrm{MPA}$ & $\mathrm{MPV}$ & $\mathrm{TPV}$ & \\
\hline CAG-A & 458 & 40 & 418 & 0.16 & 0.20 & 17.9 \\
CAG-H & 438 & 111 & 326 & 0.13 & 0.24 & 22.4 \\
CAG-HM & 496 & 100 & 396 & 0.16 & 0.27 & 21.6 \\
\hline
\end{tabular}

range of $326-418 \mathrm{~m}^{2} / \mathrm{g}$ and $0.13-0.16 \mathrm{~cm}^{3} / \mathrm{g}$. It was observed that CAG-HM, obtaining by using hexamethylenetetramine (HMTA) as the catalyst exhibited highest BET-SA of $496 \mathrm{~m}^{2} / \mathrm{g}$, which might be due to the formation of smaller cluster during the gel formation, as evidenced from the corresponding SEM image (Figure 3(c)). The BET surface area of carbon aerogels obtained by from resorcinol and formaldehyde system [11] using aqueous $\mathrm{Na}_{2} \mathrm{CO}_{3}$ as catalyst was found to be $547 \mathrm{~m}^{2} / \mathrm{g}$, which was slightly higher than that of the present system. Although the BET results were found to be slightly lower in this present study, however, the major advantage is the shortening of supercritical drying process, in which the lengthy process of solvent exchange is avoided by this new methodology and this is considered to be an important criterion for the product development. The study of gas sorption properties, especially the $\mathrm{H}_{2}$ uptake is in progress and will be reported soon elsewhere.

\section{Conclusion}

Carbon aerogels were prepared from resorcinol and furfural using three different catalysts followed by supercritical drying of IPA conditions and by pyrolysis under inert conditions. The gelation time is faster in hexame- 
thylenetetramine. Among the carbon aerogels, hexamethylenetetramine catalyzed sample shows good physical properties in terms of their surface characteristics. As per the SEM images are concerned, the carbon aerogel, obtained from hexamethylenetetramine catalyzed reaction possesses dense interconnected network of carbon particles and hence possess high surface area than that of the acid catalyzed products. Moreover, this present methodology provides an easy and simpler technique for the preparation of carbon aerogels without rigorous exchange of pore liquid, generally employed prior to the supercritical drying.

\section{Acknowledgements}

Authors gratefully acknowledged the receipt of financial assistance from Department of Electronics \& Information Technology (DeitY), and Department of Science \& Technology (DST), New Delhi for this work. K S Rejitha thanks Kerala State Council for Science Technology and Environment (KSCSTE) for the postdoctoral fellowship provided to her under the KSCSTE-PDF 2010 scheme.

\section{REFERENCES}

[1] H. Kabbour, T. F. Baumann Jr., J. H. Satcher, A. Saulnier and C. C. Ahn, "Toward New Candidates for Hydrogen Storage: High-Surface-Area Carbon Aerogels," Chemistry of Materials, Vol. 18, No. 26, 2006, pp. 6085-6087. doi:10.1021/cm062329a

[2] H. Y. Tian, C. E. Buckly, S. B. Wang and M. F. Zhou, "Enhanced Hydrogen Storage Capacity in Carbon Aerogels Treated with KOH," Carbon, Vol. 47, No. 8, 2009, pp. 2128-2130. doi:10.1016/j.carbon.2009.03.063

[3] M. Armandi, B. Boneli, I. Bottero, C. O. Arean and E. Garronem, "Synthesis and Characterization of Ordered Porous Carbons with Potential Applications as Hydrogen Storage Media," Microporous and Mesoporous Materials, Vol. 103, No. 1-3, 2007, pp. 150-157. doi:10.1016/j.micromeso.2007.01.049

[4] H. Y. Tian, C. E. Buckley, S. Mule, M. Paskevicius and B. B. Dhal, "Preparation, Microstructure and Hydrogen Sorption Properties of Nano-Porous Carbon Aerogels Under Ambient Drying," Nanotechnology, Vol. 19, No. 47, 2008. doi:10.1088/0957-4484/19/47/475605

[5] A. W. C. van den Berg and C. O. Arean, "Materials for Hydrogen Storage: Current Research Trends and Perspectives," Chemical Communications, No. 6, 2008, pp. 668-681. doi:10.1039/B712576N

[6] S. Deng, X. Xiao, L. Han, Y. Li, S. Li, H. Ge, Q. Wang and L. Chen, "Hydrogen Storage Performance of $5 \mathrm{LiBH}_{4}$ $+\mathrm{Mg}_{2} \mathrm{FeH}_{6}$ Composite System," International Journal of Hydrogen Energy, Vol. 37, No. 8, 2012, pp. 6733-6740. doi:10.1016/j.ijhydene.2012.01.094

[7] M. Shiraishi, T. Takenobu, H. Kataura and M. Ata, "Hydrogen Adsorption and Desorption in Carbon Nanotube
Systems and its Mechanisms," Applied Physics A, Vol. 78, No. 7, 2004, pp. 947-953.

doi:10.1007/s00339-003-2413-0

[8] M. Haluska, M. Harcher, M. Becher, U. Dettlaff-Wegliskowka, X. Chen and S. Roth, "Interaction of Hydrogen Isotopes with Carbon Nanostructures," Materials Science and Engineering B-Solid, Vol. 108, No. 1-2, 2004, pp. 130-133. doi:10.1016/j.mseb.2003.10.092

[9] S. Bhaumik, S. Murad and I. K. Puri, "Hydrogen Storage in Carbon Nanostructures: Possibilities and Challenges for Fundamental Molecular Simulations," Proceedings of IEEE, Vol. 94, No. 10, 2006, pp.1806-1814. doi:10.1109/JPROC.2006.883703

[10] U. Fischer, R. Selagar, V. Block, R. Petricevic and J. Frick "Carbon Aerogel as Electrode Material in Supercapacitor," Journal of Porous Materials, Vol. 4, No. 4, 1997, pp. 281-285. doi:10.1023/A:1009629423578

[11] R. A. Ganeev, P. A. Naik, J. A. Chakera, H. Singhal, N. C. Pramanik, P. A. Abraham, N. Rani Panicker, M. Kumar and P. D. Gupta, "Carbon Aerogel Plumes as an Efficient Medium for Higher Harmonic Generation in 50 - $90 \mathrm{~nm}$ Range," Journal of the Optical Society of America B, Vol. 28, No. 3, 2011, pp. 360-364. doi:10.1364/JOSAB.28.000360

[12] N. Job, A. Théry, R. Pirard, J. Marien, L. Kocon, J. Rouzaud, F. Béguin and J. P. Pirard, "Carbon Aerogels, Cryogels and Xerogels: Influence of the Drying Method on the Textural Properties of Porous Carbon Materials," Carbon, Vol. 43, No. 12, 2005, pp. 2481-2494.

doi:10.1016/i.carbon.2005.04.031

[13] H. Y. Tian, C. E. Buckley, M. Paskevcius and D. A. Sheppard, "Acetic Acid Catalyzed Carbon Xerogels Derived from Resorcinol-Furfural for Hydrogen Storage," International Journal of Hydrogen Energy, Vol. 36, No. 1, 2011, pp. 671-679. doi:10.1016/j.ijhydene.2010.10.054

[14] R. W. Pekala, "Organic Aerogel from Polycondensation of Resorcinol with Formaldehyde," Journal of Materials Science, Vol. 24, No. 9, 1989, pp. 3221-3271. doi:10.1007/BF01139044

[15] R. W. Pekala, S. T. Mayer, J. L. Kaschmitter and F. M. Kong, "Carbon Aerogels: An Update on Structure, Properties and Applications," In: Y. A. Attia, Ed., Sol-Gel Processing and Applications, Plenum Press, New York, 1994, p. 369. doi:10.1007/978-1-4651-2570-7_32

[16] D. Wu, R. Fu, S. Zhang, M. S. Dresselhaus and G. Dresselhaus, "Preparation of Low Density Carbon Aerogels by Ambient Pressure Drying," Carbon, Vol. 42, No. 10, 2004, pp. 2033-2039. doi:10.1016/j.carbon.2004.04.003

[17] D. Wu, R. Fu, S. Zhang, M. S. Dresselhaus and M. Dresselhaus, "The preparation of Carbon Aerogels Based upon the Gelation of Resorcinol-Furfural in Isopropanol with Organic Base Catalyst," Journal of Non-Crystalline Solids, Vol. 336, No. 1, 2004, pp. 26-31. doi:10.1016/j.jnoncrysol.2003.12.051

[18] R. M. Silverstein and F. X. Webster, "Spectrometric Identification of Organic Compounds," 6th Edition, John Wiley \& Sons., New York, 1998. 